\title{
Antiviral protection following immunization correlates with humoral but not cell-mediated immunity
}

\author{
Vijay Panchanathan, Geeta Chaudhri and Gunasegaran Karupiah
}

Smallpox was a deadly disease when it was rife yet despite its eradication more than 30 years ago, the possibility of accidental or intentional release has driven research in search of better definitions of correlates of protective immunity. Mousepox, a disease caused by ectromelia virus (ECTV), is arguably one of the best surrogate small animal models for smallpox. Correlates of protection in mousepox are well defined during primary infection, whereas those in a secondary infection, which have definite relevance to vaccination strategies, are less well understood. We previously established that neutralizing antibody (Ab), which is generated far more rapidly during a secondary infection compared with a primary infection, has a key role during a secondary virus challenge. In this study, we show that the route of immunization or the use of homologous or heterologous virus vaccines for immunization does not influence the ability of mice to control high-dose virulent ECTV challenge or to mount a substantial secondary neutralizing $\mathrm{Ab}$ response. In contrast, the recall cytotoxic $\mathrm{T}$ lymphocyte $(\mathrm{CTL})$ responses generated under these regimes of immunization were varied and did not correlate with virus control. Furthermore, unlike the recall Ab response that was generated rapidly, the kinetics of the secondary antiviral CTL response was no different to a primary infection and peaked only at day $\mathbf{8}$ post-challenge. This finding further underscores the importance of $\mathrm{Ab}$ in conferring protection during secondary poxvirus infection. This information could potentially prove useful in the design of safer and more efficacious vaccines against poxviruses or other diseases using poxvirus vectors.

Immunology and Cell Biology (2010) 88, 461-467; doi:10.1038/icb.2009.110; published online 12 January 2010

Keywords: antibody; cytotoxic T lympohocyte; memory; poxvirus infection protective immunity; vaccination

Smallpox was an ancient scourge, causing mortality rates as high as $30-40 \%$ in the past. ${ }^{1}$ Fortunately, this deadly disease was eradicated through the highly successful smallpox eradication campaign involving vaccination. ${ }^{1}$ Fears of an intentional or accidental release of variola virus, the causative agent of smallpox, have sparked interest in understanding the correlates of protection in smallpox. Very little is known regarding protective immunity to smallpox as the currently available tools of molecular biology, immunology and virology were not available at the time the disease was rife. Furthermore, the fact that the currently licensed vaccine for smallpox has unacceptable rates of adverse effects emphasizes not only the need to understand immunity to smallpox but also to develop effective and safer vaccination strategies. $^{2}$

In the absence of clinical cases of smallpox, surrogate animal models such as variola virus and monkeypox virus (MPXV) infection in macaques and vaccinia (VACV) and ectromelia virus (ECTV) infection in mice have been used to infer requirements for recovery and protection from smallpox. Both ECTV and variola virus are highly virulent, cause disease with high mortality rates in their respective natural hosts and share many similarities in virus biology, pathogenesis and disease. Thus, the mousepox model makes for an excellent surrogate animal model to understand the requirements for recovery and protection from smallpox. In contrast, experiments with VACV in mice and MPXV in macaques use very high doses of virus in an attempt to mimic the situation in smallpox infection. In contrast, in experiments with VACV in mice and MPXV in macaques, very high doses of virus are injected directly into the bloodstream, bypassing the initial stage of virus replication in the draining LN and primary viremia observed in natural infection. As a consequence, these protocols only model responses to the secondary viremia that normally occurs after seeding to other organs.

The requirements for recovery of mice from a primary ECTV infection are well characterized. ${ }^{3-9}$ More recently, along with others, we defined some parameters critical for recovery from a secondary ECTV infection. ${ }^{10-13}$ It has become apparent that antibody (Ab) has a critical role in recovery from secondary ECTV infection whereas the role of CD8 $\mathrm{T}$ cells has become questionable. One reason why Ab has a critical role during a secondary infection is that it is generated very rapidly even in the absence of $\mathrm{CD} 4^{+} \mathrm{T}$-cell help. ${ }^{11}$ However, whether the recall antiviral cytotoxic $\mathrm{T}$ lymphocyte (CTL) response is similarly generated rapidly is not known. It is possible that the differential requirement for humoral or cell-mediated immunity for effective virus

Infection and Immunity Group, Program in Immunology, John Curtin School of Medical Research, Australian National University, Canberra, Australian Capital Territory, Australia Correspondence: Dr G Karupiah, John Curtin School of Medical Research, Building 131, Garran Road, The Australian National University, Acton, Australian Capital Territory 0200, Australia. E-mail: Guna.Karupiah@anu.edu.au

Received 20 September 2009; revised 29 November 2009; accepted 1 December 2009; published online 12 January 2010 
control during a secondary infection is related to the type of virus (vaccine) used and/or the route of immunization.

In this study, we have investigated the kinetics of the secondary anti-ECTV CTL response to determine whether it was generated more rapidly than during a primary infection. Second, we immunized mice with avirulent strains of either homologous (the avirulent, thymidine kinase-deficient strain of ECTV Moscow, hereafter referred as ECTV$\mathrm{TK}^{-}$) or heterologous (modified VACV Ankara (MVA)) viruses through three different routes and assessed whether the recall CTL and $\mathrm{Ab}$ responses correlated with virus control. MVA is heterologus relative to ECTV as not all $\mathrm{B}$ cell and CD8 $\mathrm{T}$ cell determinants are shared. The cross-reactive $\mathrm{Ab}$ and CTL responses generated by MVA are believed to be protective against ECTV challenge.

\section{RESULTS}

Similar kinetics of the primary and secondary anti-ECTV CD8 T cell responses

We have previously shown that the recall $\mathrm{Ab}$ response to secondary ECTV infection is anamnestic and is generated more rapidly than the response during a primary infection. ${ }^{11}$ Whether the antiviral CTL response is also generated more rapidly during a secondary ECTV infection when compared with a primary infection is not known. To determine whether this was the case, groups of mice were immunized (vaccinated) with ECTV-TK ${ }^{-}$and rested for 6 months. Separate groups of age-matched mice were left unimmunized. After 6 months, mice were challenged (immunized groups) or infected (unimmunized groups) with $10^{5}$ p.f.u. plaque-purified virulent ECTV Moscow WT (ATCC VR 1374; hereafter referred to as ECTV-WT) and killed after selected time-points to determine the kinetics of the primary and secondary anti-ECTV CTL responses.

Interestingly, the kinetics of the primary and secondary anti-ECTV CTL responses were similar and the ex vivo CTL activity peaked at day 8 post-infection or post-challenge (p.c., Figure 1). This finding was unexpected and contrary to what is generally reported in some other secondary viral infections. ${ }^{14}$ The day 8 time point was therefore used to measure CTL responses and viral load in organs in all subsequent experiments.
Immunization with ECTV $\mathrm{TK}^{-}$or MVA influences the magnitude of the secondary CTL response

Immunization with the avirulent ECTV $\mathrm{TK}^{-}$protects even highly susceptible strains of mice against challenge with virulent ECTVWT. ${ }^{10,11}$ In previous studies, we had used the intraperitoneal (i.p.) route of immunization with ECTV-TK ${ }^{-}$followed by ECTV-WT for subcutaneous (s.c.) secondary challenge. We reasoned that from a vaccination standpoint, it was important to study the effect of using more appropriate routes of immunization. In this study, we have compared the i.p., s.c and intradermal (i.d.) routes of immunization. In addition, we used the highly attenuated vaccine strain of VACV, MVA, to assess whether the route of vaccination with homologous $\left(\mathrm{ECTV}^{-\mathrm{TK}^{-}}\right.$) or heterologous (MVA) virus influenced the magnitude of recall CTL responses. Figure 2 shows the ex vivo CTL activity

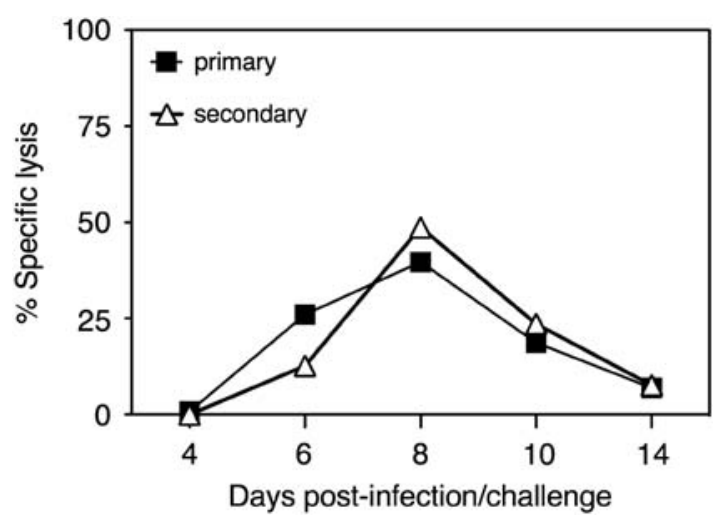

Figure 1 CTL activity in primary and secondary ECTV infections. Groups of female mice were either unimmunized or immunized via the i.p. route with ECTV TK- and 6 months later infected or challenged with ECTV-WT. At various times post-infection (p.i.) or p.c. the mice were killed and splenocytes obtained from five mice in each group at each time point pooled and splenic ECTV-specific CTL activity was measured ex vivo using ECTVWT-infected targets. Data shown are percentage-specific lysis at an effector:target ratio of 100:1. Data are representative of two separate experiments.

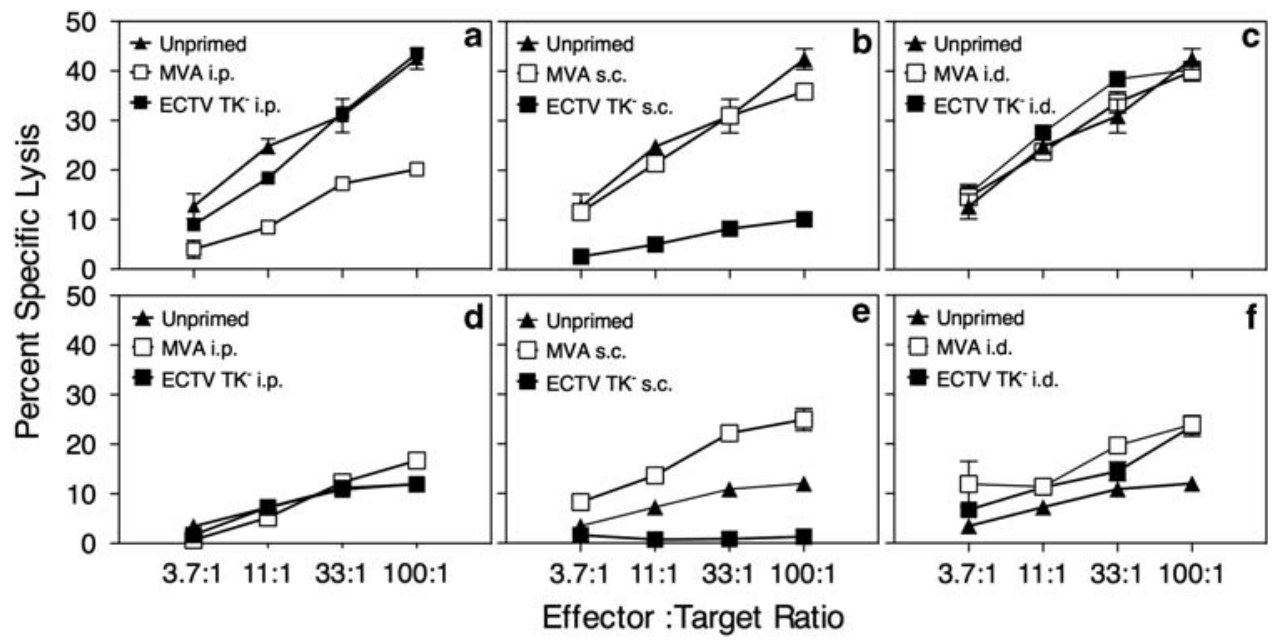

Figure 2 Effect of route of immunization or vaccine type on recall CTL responses. Groups of female mice were either unimmunized or immunized via the i.p., s.c. or i.d. routes with either ECTV TK- or MVA and 6 months later challenged with ECTV-WT. At day 8 p.c., the mice were killed and splenocytes obtained from five mice in each group were pooled and splenic ECTV-specific CTL activity was measured ex vivo using ECTV-infected (a, $\mathbf{b}$ and $\mathbf{c}$ ) or EVM163 $20-27$ peptide-pulsed (d, e and f) targets and uninfected MC57G target cells. Data shown are representative of two separate experiments. 
against syngeneic MC57G targets infected with ECTV (Figures 2a, b and c) and the cytolytic response against targets pulsed with the EVM163- (or B8R)-derived TSYKFESV peptide (Figures 2d, e and f). Consistent with our published results, ${ }^{10,11}$ mice immunized i.p. with ECTV-TK ${ }^{-}$generated a robust secondary CTL response 8 days p.c, approximating the levels of cytolysis observed during primary ECTV infection in unprimed mice (Figure 1). ${ }^{4}$ However, the recall response in animals immunized i.p. with MVA was weaker (Figure 2a). Conversely, the recall CTL response in mice given MVA via the s.c. route of immunization was 27-fold higher in magnitude compared with responses in mice immunized s.c. with ECTV-TK ${ }^{-}$(Figure 2b). The CTL activity of the MVA-s.c.-immunized mice was also similar to the activity in unimmunized, ECTV-infected mice. In contrast, CTL responses in groups immunized with MVA or with ECTV-TK ${ }^{-}$via the i.d. route were comparable and similar to responses in unimmunized, ECTV-infected mice (Figure 2c). No CTL activity was detected after ECTV challenge in any of the vaccinated groups at day 4 p.c. (data not shown), consistent with the result in Figure 1 that the secondary CTL response was not generated more rapidly than a primary response.

To determine whether the differences in the total antiviral CTL responses were mirrored by viral determinant-specific response, the immunodominant CD8 $\mathrm{T}$ cell determinant peptide (TSYKFESV) of VACV/ECTV was used to pulse targets in a CTL assay. This determinant $\mathrm{B}_{8 \mathrm{R}_{20-27}}$ (encoded by the open reading frame $\mathrm{B} 8 \mathrm{R}$ in VACV) is present in both MVA and ECTV (encoded by the EVM163 open reading frame) and is conserved within members of the orthopoxviridae. ${ }^{15}$ The differences observed in the total anti-ECTV CTL response in mice immunized with either vaccine via the i.p. route (Figure 2a) did not translate to any differences in the response to the EVM163 determinant (Figure 2d). The i.d. route of immunization with MVA or with ECTV-TK ${ }^{-}$also resulted in similar responses to the EVM163 determinant. It is noteworthy that the complete absence of any response to EVM163 peptide-pulsed targets in mice primed s.c. with ECTV-TK ${ }^{-}$virus (Figure 2e). This is in contrast to mice primed s.c. with MVA, which generated a potent response (Figure 2e). Thus, the type of virus used and the route of vaccination clearly influenced the magnitude of the recall antiviral CTL responses.

\section{Effect of routes of immunization on determinant-specific CD8 $\mathrm{T}$ cell numbers}

Next we used EVM163 $20-27$ peptide conjugated-MHC class I $\mathrm{K}^{\mathrm{b}}$ tetramers to assess whether the differences in magnitude observed in the secondary CTL responses were reflected in the numbers of antigen-specific splenic $\mathrm{CD}^{+} \mathrm{T}$ cells using flow cytometry (Figures $3 \mathrm{a}$ and $\mathrm{b}$ ). As depicted in Figure $3 \mathrm{c}$ and Table 1, the percentage of tetramer ${ }^{+} \mathrm{CD} 8 \mathrm{~T}$ cells were similar in mice vaccinated with MVA via the i.p. or s.c. routes (2.81 and $3.35 \%$ of $\mathrm{CD}^{+} \mathrm{T}$ cells, respectively) but at least two- to threefold lower in the group vaccinated via the i.d. route $\left(1.73 \%\right.$ of $\mathrm{CD}^{+} \mathrm{T}$ cells). It is noteworthy that numbers of tetramer ${ }^{+}$cells are significantly lower than those generated during a primary ECTV infection $\left(6.5 \%\right.$ of $\mathrm{CD}^{+} \mathrm{T}$ cells in a primary infection

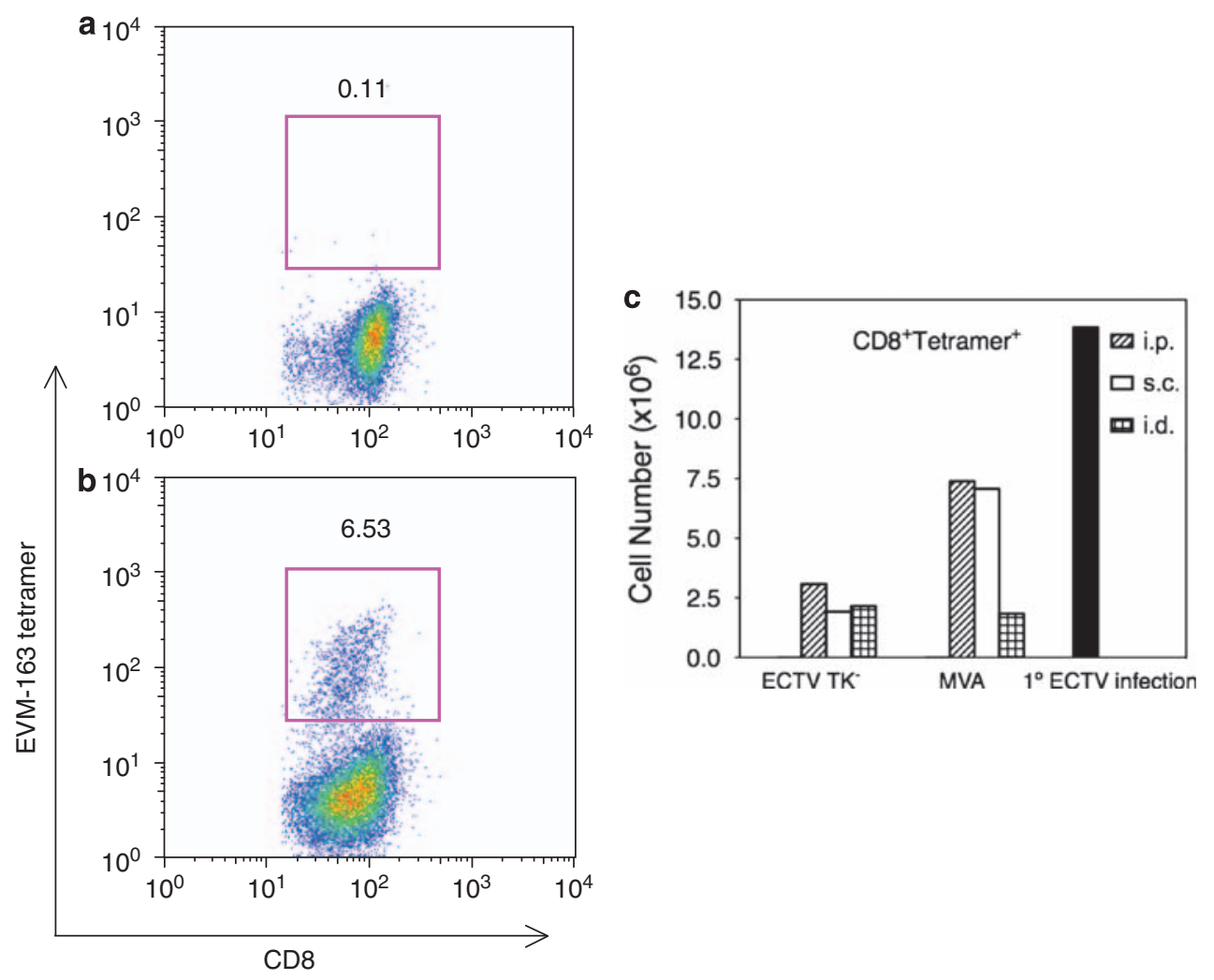

Figure 3 Enumeration of EVM163 $20-27$-specific CD8 T cells. Groups of female mice were immunized, challenged and killed as in Figure 2. Splenocytes obtained from five mice in each group were pooled and flow cytometry performed to enumerate EVM163 $20-27$-specific CD8 T cells as described in Materials and Methods section. (a) Percentage of $\mathrm{CD}^{+} \mathrm{T}$ cells that are also tetramer ${ }^{+}$in naïve C57BL/6 mice. Whole splenocytes were first gated on lymphocytes and next on $\mathrm{CD} 8^{+} \mathrm{T}$ cells. (b) Percentage of $\mathrm{CD} 8^{+} \mathrm{T}$ cells that are also tetramer ${ }^{+}$in C57BL/6 mice 8 days after primary infection with ECTV. Data shown are percentage of $\mathrm{CD}^{+} \mathrm{T}$ cells that stain positive with the EVM163 $20-27$ tetramer. (c) Data shown are numbers of EVM163 20-27-Specific CD8 T cells per spleen of pooled splenocytes for groups primed through various routes with MVA or ECTV TK- ${ }^{-}$. Data shown are representative of two separate experiments. 
Table 1 EVM-163 tetramer-specific CD8+ T cells

\begin{tabular}{llccc}
\hline $\begin{array}{l}\text { Immunization/ } \\
\text { infection }\end{array}$ & $\begin{array}{l}\text { Route of } \\
\text { immunization }\end{array}$ & $\begin{array}{c}\% C D 8^{+} \\
\text {Tcells }\end{array}$ & $\begin{array}{c}\text { \%EVM163 } \\
\text { tetramer } \\
\text { cells }\end{array}$ & $\begin{array}{c}\text { \# EVM163 } \\
\text { tetramer cells }^{+}\end{array}$ \\
\hline ECTV TK- & i.p. & 15.3 & 3.12 & $3.07 \times 10^{6}$ \\
ECTV TK- & s.c. & 11.3 & 1.46 & $1.92 \times 10^{6}$ \\
ECTV TK- & i.d. & 24.2 & 3.57 & $2.16 \times 10^{6}$ \\
MVA & i.p. & 13.1 & 2.81 & $7.39 \times 10^{6}$ \\
MVA & s.c. & 37.3 & 3.35 & $7.07 \times 10^{6}$ \\
MVA & i.d. & 10.6 & 1.73 & $1.84 \times 10^{6}$ \\
Primary ECTV & Nil & 26.9 & 6.53 & $1.48 \times 10^{7}$ \\
infection $^{\text {b }}$ & & & &
\end{tabular}

Abbreviations: ECTV, ectromelia virus; i.d., intradermal; i.p., intraperitoneal; MVA, modified VACV Ankara; s.c., subcutaneous.

aThis group was infected via the s.c. route with ECTV-WT.

balues are expressed as a percentage of $\mathrm{CD} 8^{+}$cells in the spleen.

versus $<3.6 \%$ in secondary challenge). In contrast, the route of vaccination with ECTV-TK ${ }^{-}$had no influence on the numbers of tetramer ${ }^{+} \mathrm{CD}^{+} \mathrm{T}$ cells generated after virulent ECTV challenge (Figure 3c). Taken together, the data shown in Figures 2 and 3 indicated that the type of virus used and the route of immunization clearly influence the magnitude of the recall CTL response but there was no correlation with numbers of antigen-specific $\mathrm{CD} 8^{+} \mathrm{T}$ cells. This suggested that the vaccine type and the routes of immunization influence both the quantitative and qualitative aspects of the antiviral $\mathrm{CD}^{+}{ }^{+} \mathrm{T}$-cell response.

\section{Route of immunization has no effect on virus control}

Liver damage and liver failure, as a result of uncontrolled ECTV replication within the organ, is a major reason for mortality in mousepox $^{16-18}$ We, therefore, measured viral load in livers from vaccinated and non-vaccinated (unimmunized) mice that had been challenged with ECTV. Interestingly, the route of immunization or the type of virus used to immunize mice did not influence their capacity to control viral load in the liver (Figure 4). Virus control in all groups of vaccinated mice was comparable, and as expected, vaccinated mice controlled virus replication more effectively than non-vaccinated mice at day 8 p.c.

\section{Effect of immunization with ECTV $\mathrm{TK}^{-}$and MVA on the secondary Ab response}

As the varied levels of CTL activity in the different groups could not possibly account for the comparable levels of virus control, we analyzed the potential contribution of humoral immunity. ECTVspecific IgG levels were similar in all groups irrespective of the route of immunization, albeit, absorbance levels were marginally higher in ECTV-TK ${ }^{-}$-immunized mice (Figures $5 \mathrm{a}$ and $\mathrm{b}$ ). However, because ECTV-specific IgG levels alone do not necessarily correlate with virusneutralizing Ab titers, ${ }^{10,11}$ sera were assessed for virus-neutralizing activity by the plaque reduction neutralization test. Figures $5 \mathrm{c}$ and $\mathrm{d}$ show that regardless of the route of immunization or the type of virus used, all groups of mice generated a neutralizing $\mathrm{Ab}$ response on day 8 p.c. In agreement with our previous finding, ${ }^{10,11}$ mice immunized i.p. with ECTV $\mathrm{TK}^{-}$generated high virus-neutralizing $\mathrm{Ab}$ titers in response to virulent ECTV challenge (Figure 5d). Mice immunized with $\mathrm{ECTV} \mathrm{TK}^{-}$via the s.c. or i.d. routes had similar titers but were approximately a third of the values of the group immunized via the i.p route. In the case of MVA-immunized mice, the s.c. route generated neutralizing $\mathrm{Ab}$ titers that were approximately twofold higher than the i.p. or i.d. routes (Figure $5 d$ ).

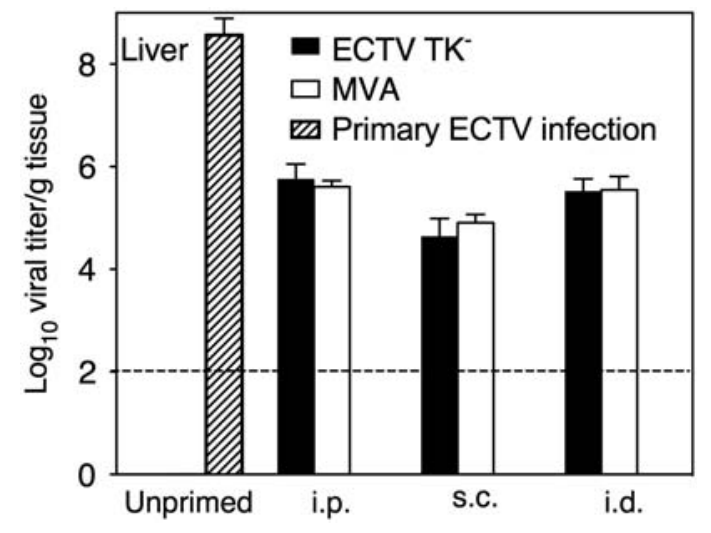

Figure 4 Effect of route of immunization or vaccine type on viral load in liver after secondary ECTV challenge. Groups of mice were either unprimed or primed via the i.p., i.d. or s.c. routes with ECTV TK $^{-}$or MVA and 6 months later challenged with ECTV-WT. At day 8 p.c., mice were killed and organs collected aseptically for determination of viral load. For comparison, viral load in unprimed mice is shown. The broken line indicates the limit of detection of the assay, which for these experiments is 100 p.f.u. Data shown are mean \pm s.e.m. from five mice in each group and are representative of two separate experiments.

We next analyzed whether the neutralizing Ab levels correlated with B-cell memory and germinal center (GC) formation. ${ }^{19}$ This was assessed through detection of GL7 and Fas expression on B cells by flow cytometry. ${ }^{20}$ In the MVA-immunized group, the numbers of splenic GC B cells $\left(\mathrm{GL}^{+} \mathrm{Fas}^{+}\right)$were higher by approximately three- to fourfold in groups immunized via the i.p. or s.c. routes compared with mice immunized via the i.d. route (Figure 5e). In contrast, the numbers of GC B cells were similar in all groups immunized with ECTV TK ${ }^{-}$, regardless of the route of immunization (Figure $5 \mathrm{f}$ ). The data indicated a lack of correlation between neutralizing Ab detected at day 8 with formation of new GC, implying a role for extrafollicular plasma cells in the generation of neutralizing $\mathrm{Ab}$, at least during the first 8 days p.c.

\section{DISCUSSION}

We have previously shown that immunization with the avirulent ECTV $\mathrm{TK}^{-}$protected mice from virulent ECTV-WT challenge and that protection was associated with a neutralizing Ab response. ${ }^{10,11}$ Mice deficient in type I and II interferon, $\mathrm{CD}^{+} \mathrm{T}$ cells or effector molecules such as perforin and granzymes $\mathrm{A}$ and $\mathrm{B}$ that had been immunized with ECTV $\mathrm{TK}^{-}$were able to control virus as effectively as B6 wild-type (WT) mice after a secondary challenge. In addition, ECTV $\mathrm{TK}^{-}$immunized B6 WT mice depleted of CD4 and/or CD8 T cells at the time of challenge were also able to control secondary ECTV-WT infection and generated high titers of virus-neutralizing Ab. In those studies, the i.p. route of immunization and a sub-lethal challenge dose of virus were used. In this study, we have assessed the effect of using different virus types and routes for immunization.

Regardless of the route of immunization with either homologous ECTV $\mathrm{TK}^{-}$or heterologous MVA, viral load in the liver after virulent ECTV challenge was similar but significantly $(P<0.05)$ lower than titers in unimmunized mice indicating that the route of immunization did not ultimately influence virus control (Figure 4). This is an important finding, particularly in the case of the MVA vaccine, as even at the sub-optimal dose used for immunization, virus control was still effective.

Despite the lack of any significant differences in viral load when either MVA or ECTV-TK ${ }^{-}$was used for immunization through three 

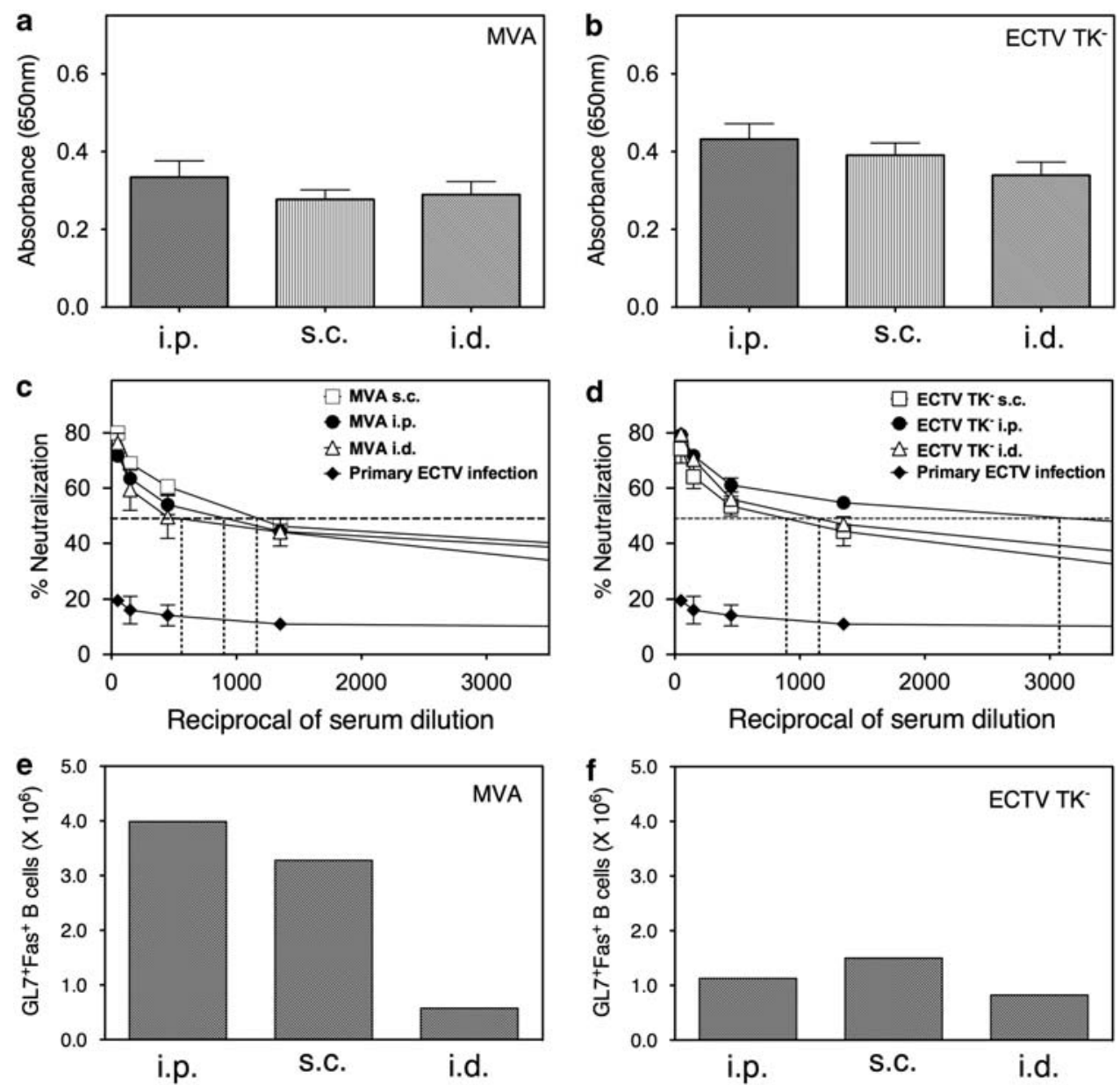

Figure 5 ECTV-specific IgG, neutralizing Ab and GC responses. Groups of mice were immunized, challenged and killed as described in Figure 2. Sera were used to measure the ECTV-specific IgG levels (absorbance) at a dilution of 1/100 in MVA-primed (a) and ECTV TK- ${ }^{-}$primed (b) mice. The neutralizing Ab response from MVA-primed (c) or ECTV TK ${ }^{-}$-primed (d) mice was determined by the plaque reduction neutralization test (PRNT). The neutralizing titers for day 8 during a primary ECTV infection are shown for comparison. Data shown are means \pm s.e.m. of the neutralizing titers of five individual serum samples from each group. Splenocytes obtained from each group on day 8 p.c. were pooled and assessed by flow cytometry for GC markers (GL7 and Fas), comparing MVA-primed (e) and ECTV TK ${ }^{-}$-primed (f) mice. Data shown are representative of two separate experiments.

different routes, there were clear differences in the magnitude of the recall CTL responses (Figure 2). The s.c. and i.d. routes of immunization with MVA generated potent recall CTL responses to ECTV and the EVM163 $20-27$ determinant, whereas the i.p. route generated a weaker response. In contrast, although the i.d. and i.p. routes of immunization with $\mathrm{ECTV}_{-} \mathrm{TK}^{-}$resulted in strong recall CTL responses, the s.c. route of immunization resulted in weak secondary CTL responses. The differences in the magnitude of the recall CTL responses did not appear to be related to numbers of antigen-specific cells, as there was a lack of correlation between the numbers of EVM163 $20-27$ tetramer ${ }^{+} \mathrm{CD}^{+} \mathrm{T}$ cells and the levels of lysis of EVM163 $20-27$ peptide-pulsed targets (Figures 2 and 3). At least two key points could be made in relation to this finding. First, it suggests the possibility that $\mathrm{CD} 8^{+} \mathrm{CTL}$ of differing avidity are generated by immunization with the different viruses and/or routes. In fact, there is evidence that the ability to kill virus-infected targets in vitro is not predictive of in vivo efficacy. ${ }^{21}$ Further, T cells with identical specificities could have different avidities, which again influence their function. ${ }^{22}$ It has also been shown that that high-avidity CTL are more effective in elimination of infected cells in vivo. ${ }^{23}$ Second, at least in the ECTV model, an antiviral CTL response may not be critical for viral control during a secondary infection.
We have previously shown that perforin-mediated cytolytic activity of $\mathrm{CD}^{+} \mathrm{T}$ cells is essential for ECTV control in a primary ECTV infection. ${ }^{11,24,25}$ Thus, the lack of any correlation between the magnitude of CTL activity and virus load or our finding that the kinetics and magnitude of secondary anti-ECTV CTL responses are similar to those in a primary infection does not support a role for memory CD8 T cell response in curtailing viral load during a secondary infection (Figure 1). Nevertheless, there is a possibility that anti-ECTV CD8 ${ }^{+}$ T cells may use non-cytolytic effector mechanisms to control virus, which would imply that the magnitude of the cytolytic activity may not be relevant. We believe this is unlikely as the data presented here are consistent with our published work, which indicated that the absence of CD8 T cells or the CD8 T cell effector molecules granzyme A, granzyme $B$, perforin or interferon- $\gamma$ during a secondary viral challenge did not affect virus clearance. ${ }^{11}$

Our results from the kinetic study may seem at odds with the current dogma, in which the memory $\mathrm{CD} 8^{+} \mathrm{T}$-cell response is believed to be usually more rapid and robust in comparison with the primary response. ${ }^{14,26-29}$ At least two key factors may account for the differences between those studies and ours. First, the assessment of primary and secondary responses was undertaken using TCR transgenic CD8 ${ }^{+}$ $T$ cells specific for non-viral antigens using adoptive transfer systems ${ }^{27}$ 
or a single viral antigenic determinant studied in isolation. ${ }^{28}$ Second, the studies relied on the fact that the surface glycoproteins of influenza A viruses used for infection and subsequent challenge are sufficiently different to prevent cross-neutralization by $\mathrm{Ab}$, while they share internal components that provide peptides recognized by influenzaspecific CTL. ${ }^{26}$ In our study, we have used heterologous MVA or homologous ECTV for immunization and challenged with virulent ECTV-WT. This approach will no doubt generate both cell-mediated and humoral immunity, which occur as a consequence of natural infection or after vaccination. Thus, it is possible that a need for recall CTL response to control virus is obviated in the presence of neutralizing $\mathrm{Ab}$. It is also possible that antiviral CTL do not have a role in virus control during secondary poxvirus infections.

Unlike the CTL response, the $\mathrm{Ab}$ response during a secondary ECTV infection is anamnestic and is detectable even at day 5 p.c. ${ }^{11}$ In this study, there was a strong correlation between virus control and the neutralizing $\mathrm{Ab}$ response, albeit, with a two to threefold difference in the neutralizing titers between groups. Importantly, regardless of the route of immunization or the type of virus used, sera from all groups of immunized mice had demonstrable levels of virus neutralizing $\mathrm{Ab}$ titers in response to virulent ECTV challenge.

Analysis of GC formation, quantified by the number of $\mathrm{GL}^{+} \mathrm{Fas}^{+} \mathrm{B}$ cells, showed little or no correlation between neutralizing Ab levels (Figures $5 \mathrm{c}$ and $\mathrm{d}$ ) and numbers of GC B cells (Figures $5 \mathrm{e}$ and $\mathrm{f}$ ). As an example, although neutralizing $\mathrm{Ab}$ titers were highest in mice immunized with ECTV-TK ${ }^{-}$via the i.p. route, the numbers of GC B cells were three- to fourfold lower compared with mice immunized with MVA via the i.p. or s.c. routes (Figures $5 \mathrm{e}$ and $\mathrm{f}$ ). Thus, although the secondary $\mathrm{Ab}$ response is anamnestic in nature, our data suggest that formation of new GC may not be necessary and that the extrafollicular plasma cells likely produce neutralizing $\mathrm{Ab}$ to control virus replication. Indeed, this finding is consistent with our previous study ${ }^{11}$ in which acute depletion of CD4 T cells with anti-CD4mAb, known to abolish the GC response, did not result in an increase ECTV titers or affect neutralizing Ab responses measured at day 8 p.c. Thus, regardless of vaccine type or route of inoculation, it is possible that a threshold of neutralizing $\mathrm{Ab}$ response generated by extrafollicular plasma cells restricts viral replication during the acute phase of a secondary infection.

Our findings on the importance of the humoral response in secondary ECTV infection corroborate those of Lustig et al. ${ }^{30}$ who showed that vaccinia immunoglobulin protects against VACV and ECTV challenge. In addition, another similar study showed that therapeutic post-exposure immunization with VACV-Lister and MVA conferred protection from ECTV challenge and that this protection was associated with the generation of poxvirus-specific Abs. ${ }^{30}$ In studies with other poxviruses, mice immunized with MVA were found to develop anti-poxvirus Ab responses ${ }^{31}$ and were also able to elicit T-cell responses a month after primary vaccination. ${ }^{32}$ Vaccination with two sequential doses of MVA or one dose of MVA followed by the Dryvax vaccine was able to elicit Abbinding and neutralizing titers and T-cell responses equivalent or higher than those induced by Dryvax alone after MPXV challenge, leading to healthy and asyptomatic animals, whereas unimmunized animals became very ill or died after challenge with MPXV. ${ }^{33}$ Data from another MPXV study show that MVA alone or in combination with Dryvax could elicit neutralizing $\mathrm{Ab}$ and $\mathrm{T}$-cell responses superior to Dryvax alone and these responses protected against a lethal challenge with MPXV. ${ }^{34}$ Two other studies lend support to the role of antibodies, one in a MPXV model ${ }^{35}$ and another in a VACV model. ${ }^{36}$ Although our study confirms the effectiveness of MVA as a vaccine, it is the first study to show that the use of sub-optimal doses of MVA through different routes of immunization can confer protection against a virulent ECTV challenge.
In conclusion, we have shown that vaccination with MVA or ECTV through three different routes can confer mice comparable levels of protection against virulent challenge. This ability to control virus was not associated with CTL activity but strongly correlated with the ability of mice to generate a neutralizing $\mathrm{Ab}$ response, highlighting the importance of the humoral arm of the adaptive immune response in control of virulent ECTV challenge. It is noteworthy that the finding that the $\mathrm{Ab}$ response to secondary virus challenges is generated far more rapidly than during a primary infection. This is also consistent with a role for $\mathrm{Ab}$ in virus control early during the course of a secondary infection. In contrast, the kinetics of anti-ECTV CTL response during a secondary challenge is no different to that of a primary response, consistent with the notion that $\mathrm{CD} 8^{+} \mathrm{T}$ cells are not critical for virus control during a secondary infection. ${ }^{11}$ In this regard, the best correlate of long-term protective immunity conferred by effective vaccines is neutralizing $\mathrm{Ab}$. Indeed, humoral immunity to smallpox and several other diseases in vaccinated individuals is stable and can last for decades, whereas memory CD4 and CD8 T cell responses wane more rapidly. ${ }^{37-39}$ Future studies on vaccination strategies against poxviruses or those using poxviruses as vaccine vectors should therefore ideally focus on key parameters, including dose and route of immunization, necessary for the generation and long-term maintenance of the neutralizing $\mathrm{Ab}$ response.

\section{METHODS}

\section{Viruses}

ECTV-WT and ECTV-TK ${ }^{-40}$ were propagated in BS-C-1 cells (ATCC CCL26) as described earlier. ${ }^{41}$ Modified VACV Ankara strain (MVA), kindly provided by Dr Bernard Moss, ${ }^{31}$ was grown in BHK-21 cells and used after purification.

\section{Mice}

Female, specific pathogen-free C57BL/6 (B6) mice were used between 8 and 12 weeks of age. All experiments were performed according to institutional guidelines for animal care and use.

\section{Immunization and infection}

To study the effect of route of immunization and vaccine type on recall CTL and neutralizing $\mathrm{Ab}$ responses to ECTV, groups of $\mathrm{B} 6$ mice were immunized via the i.p., i.d. or s.c. routes with either $10^{5}$ p.f.u. of ECTV-TK ${ }^{-}$or $10^{6}$ p.f.u. of MVA. The i.d. vaccination was carried out by scarification at the base of the tail and a $10 \mu \mathrm{l}$ volume of virus inoculum was applied directly onto the scars and allowed to adsorb into the base of the tail. These doses were selected based on previous studies ${ }^{11,42,43}$ to ensure that mice were challenged at a time when the there was no detectable neutralizing Ab. A control group of mice was not immunized and used for determination of primary CTL and Ab responses. After 6 months, mice were challenged s.c. with a sublethal dose of $10^{5}$ p.f.u. of ECTV-WT and killed 8 days later. Sera were used to determine neutralizing Ab titers by plaque reduction neutralization test, organs were used for determination of viral titers and portions of spleens from each animal within a group were pooled and splenocytes used in the ${ }^{51} \mathrm{Cr}$-release assay to measure CTL activity and leukocyte phenotype by flow cytometry.

\section{Enzyme-linked immunosorbent assay}

Serum samples were assayed by enzyme-linked immunosorbent assay for total ECTV-specific IgG and IgG subtypes as described earlier. ${ }^{4}$ The enzyme-linked immunosorbent assay used here predominantly detects $\mathrm{Ab}$ directed against the intracellular mature virus. Briefly, U-bottom 96-well plates (Immulon 2, Dynatech Lab Inc., Alexandria, VA, USA) were coated with purified ECTV. Sera were assayed at various dilutions (ranging from 1:50 to 1:1000) and ECTVspecific $\mathrm{Ab}$ detected using horseradish peroxidase-conjugated goat anti-mouse IgG (Southern Biotechnology Associates, Birmingham, AL, USA) and color developed with TMB One-Step substrate (Dako Cytomation, Carpinteria, CA, USA). On the basis of this, a serum dilution of 1:100 was selected for determination of relative $\mathrm{Ab}$ levels. 


\section{Plaque reduction neutralization test}

The plaque reduction neutralization test, used to determine the neutralizing activity of the Ab present in serum samples to ECTV-WT has been described. ${ }^{10}$ The neutralization titer was taken as the reciprocal of the dilution of sera that caused a $50 \%$ reduction in the number of virus plaques over and above the number of plaques in the samples with sera from naive mice. In this instance the neutralization titer measures the ability of serum to neutralize mature virus.

\section{Flow cytometry}

The following anti-mouse $\mathrm{mAbs}$ were purchased from BD Biosciences: antiCD44 (PE), anti-CD62L (PE), anti-CD127 (APC), anti-GL-7 (PE), anti-Fas (FITC), anti-B220 (PerCP) and anti-CD8 (APC). PE-conjugated MHC class I $K^{b}$-restricted CD8 $\mathrm{T}$ cell tetramers specific for the immunodominant CD8 T cell determinant peptide (TSYKFESV) of VACV (B8R ${ }_{20-27}$ encoded by B8R), which is also present in MVA and ECTV (EVM163 ${ }_{20-27}$ encoded by EVM163),${ }^{15}$ were synthesized by ACRF Biomolecular Resources Facility (BRF) (John Curtin School of Medical Research, ANU, Canberra, Australia), JCSMR. Total events for cells were acquired using a FACSort flow cytometer (BD Biosciences, San Jose, CA, USA) and analyzed using FlowJo software (Tree Star, Inc., Ashland, OR, USA).

\section{CTL assay}

CTL assays to determine the ability of splenocytes to kill ${ }^{51} \mathrm{Cr}$-labeled uninfected, virus-infected or EVM163 $20-27\left({\left.\mathrm{~B} 8 \mathrm{R}_{20-27}\right)}\right.$ peptide-pulsed syngeneic (MC57G) target cells were performed as described elsewhere. ${ }^{4}$ The EVM163 $20-27$ peptide was used at a final concentration of $10 \mathrm{nM}$.

\section{Statistical analysis}

For comparison of viral titers, the non-parametric Mann-Whitney test was used, using the statistical program GraphPad Prism (GraphPad Software, Inc., San Diego, CA, USA). A $P$-value of $<0.05$ was taken to be significant.

\section{ACKNOWLEDGEMENTS}

This work was supported by Project grants to GK and GC from the National Health and Medical Research Council (NHMRC) of Australia, an RO1 grant to GK from the National Institutes of Health (R01AI067401), an International Research Scholars Special Initiative grant on Infectious Diseases to GK from the Howard Hughes Medical Institute and a Senior Research Fellowship (NHMRC) to GK.

1 Fenner F, Henderson DA, Arita I, Jezek A, Ladnyi ID. Smallpox and Its Eradication. World Health Organization: Geneva, 1988.

2 Fulginiti VA, Papier A, Lane JM, Neff JM, Henderson DA. Smallpox vaccination: review, part II. Adverse events. Clin Infect Dis 2003; 37: 251-271.

3 Chaudhri G, Panchanathan V, Bluethmann H, Karupiah G. Obligatory requirement for antibody in recovery from a primary poxvirus infection. J Virol 2006; 80: 6339-6344.

4 Chaudhri G, Panchanathan V, Buller RM, van den Eertwegh AJ, Claassen E, Zhou J et al. Polarized type 1 cytokine response and cell-mediated immunity determine genetic resistance to mousepox. Proc Natl Acad Sci USA 2004; 101: 9057-9062.

5 Karupiah G. Type 1 and type 2 cytokines in antiviral defense. Vet Immunol Immunopathol 1998; 63: 105-109.

6 Karupiah G, Buller RM, Van Rooijen N, Duarte CJ, Chen J. Different roles for CD4+ and CD8+ T lymphocytes and macrophage subsets in the control of a generalized virus infection. J Virol 1996; 70: 8301-8309.

7 Karupiah G, Chen JH, Nathan CF, Mahalingam S, MacMicking JD. Identification of nitric oxide synthase 2 as an innate resistance locus against ectromelia virus infection. J Virol 1998; 72: 7703-7706.

8 Karupiah G, Fredrickson TN, Holmes KL, Khairallah LH, Buller RM. Importance of interferons in recovery from mousepox. J Virol 1993; 67: 4214-4226.

9 Fang M, Sigal LJ. Antibodies and CD8+ T cells are complementary and essential for natural resistance to a highly lethal cytopathic virus. J Immunol 2005; 175: 6829-6836.

10 Panchanathan V, Chaudhri G, Karupiah G. Interferon function is not required for recovery from a secondary poxvirus infection. Proc Natl Acad Sci USA 2005; 102: 12921-12926.

11 Panchanathan V, Chaudhri G, Karupiah G. Protective immunity against secondary poxvirus infection is dependent on antibody but not on CD4 or CD8 T-cell function. J Virol 2006; 80: 6333-6338.

12 Fang M, Cheng H, Dai Z, Bu Z, Sigal LJ. Immunization with a single extracellular enveloped virus protein produced in bacteria provides partial protection from a lethal orthopoxvirus infection in a natural host. Virology 2006; 345: 231-243.
13 Xu RH, Fang M, Klein-Szanto A, Sigal LJ. Memory CD8+ T cells are gatekeepers of the lymph node draining the site of viral infection. Proc Natl Acad Sci USA 2007; 104: 10992-10997.

14 Ahmed R, Gray D. Immunological memory and protective immunity: understanding their relation. Science 1996; 272: 54-60.

15 Tscharke DC, Karupiah G, Zhou J, Palmore T, Irvine KR, Haeryfar SM et al. Identification of poxvirus $\mathrm{CD} 8+\mathrm{T}$ cell determinants to enable rational design and characterization of smallpox vaccines. J Exp Med 2005; 201: 95-104.

16 Esteban DJ, Buller RM. Ectromelia virus: the causative agent of mousepox. J Gen Virol 2005; 86: 2645-2659.

17 Fenner F. Mouse-pox; infectious ectromelia of mice; a review. J Immunol 1949; 63: 341-373.

18 Roberts JA. Growth of ectromelia virus in the liver parenchymal cells of different strains of mouse. Nature 1964; 202: 1140-1141.

19 Gray D, Dullforce P, Jainandunsing S. Memory B cell development but not germinal center formation is impaired by in vivo blockade of CD40-CD40 ligand interaction. $J$ Exp Med 1994; 180: 141-155.

20 Han S, Zheng B, Takahashi Y, Kelsoe G. Distinctive characteristics of germinal center B cells. Semin Immunol 1997; 9: 255-260.

21 Alexander-Miller MA, Leggatt GR, Berzofsky JA. Selective expansion of high- or lowavidity cytotoxic $T$ lymphocytes and efficacy for adoptive immunotherapy. Proc Natl Acad Sci USA 1996; 93: 4102-4107.

22 Hofmann M, Radsak M, Rechtsteiner G, Wiemann K, Günder M, Bien-Gräter U et al. T cell avidity determines the level of CTL activation. Eur J Immunol 2004; 34: 1798-1806.

23 Derby M, Alexander-Miller M, Tse R, Berzofsky J. High-avidity CTL exploit two complementary mechanisms to provide better protection against viral infection than low-avidity CTL. J Immunol 2001; 166: 1690-1697.

24 Chaudhri G, Quah BJ, Wang Y, Tan AH, Zhou J, Karupiah G et al. T cell receptor sharing by cytotoxic T lymphocytes facilitates efficient virus control. Proc Natl Acad Sci USA 2009. 106: 14984-14989.

25 Ramshaw IA, Ramsay AJ, Karupiah G, Rolph MS, Mahalingam S, Ruby JC. Cytokines and immunity to viral infections. Immunol Rev 1997; 159: 119-135.

26 Belz GT, Xie W, Doherty PC. Diversity of epitope and cytokine profiles for primary and secondary influenza a virus-specific CD8+ T cell responses. J Immunol 2001; 166: 4627-4633.

27 Cho BK, Wang C, Sugawa S, Eisen HN, Chen J. Functional differences between memory and naive CD8T cells. Proc Natl Acad Sci USA 1999; 96: 2976-2981.

28 Slifka MK, Whitton JL. Activated and memory CD8+ T cells can be distinguished by their cytokine profiles and phenotypic markers. J Immunol 2000; 164: 208-216.

29 Veiga-Fernandes H, Walter U, Bourgeois C, McLean A, Rocha B. Response of naive and memory CD8+ T cells to antigen stimulation in vivo. Nat Immunol 2000; 1: 47-53.

30 Lustig S, Maik-Rachline G, Paran N, Melamed S, Israely T, Erez N et al. Effective postexposure protection against lethal orthopoxviruses infection by vaccinia immune globulin involves induction of adaptive immune response. Vaccine 2009; 27: 1691-1699.

31 Wyatt LS, Earl PL, Eller LA, Moss B. Highly attenuated smallpox vaccine protects mice with and without immune deficiencies against pathogenic vaccinia virus challenge. Proc Natl Acad Sci USA 2004; 101: 4590-4595

32 Belyakov IM, Earl P, Dzutsev A, Kuznetsov VA, Lemon M, Wyatt LS et al. Shared modes of protection against poxvirus infection by attenuated and conventional smallpox vaccine viruses. Proc Natl Acad Sci USA 2003; 100: 9458-9463.

33 Earl PL, Americo JL, Wyatt LS, Eller LA, Whitbeck JC, Cohen GH et al. Immunogenicity of a highly attenuated MVA smallpox vaccine and protection against monkeypox. Nature 2004; 428: 182-185.

34 Stittelaar KJ, van Amerongen G, Kondova I, Kuiken T, van Lavieren RF, Pistoor FH et al. Modified vaccinia virus Ankara protects macaques against respiratory challenge with monkeypox virus. J Virol 2005; 79: 7845-7851.

35 Edghill-Smith Y, Golding H, Manischewitz J, King LR, Scott D, Bray M et al. Smallpox vaccine-induced antibodies are necessary and sufficient for protection against monkeypox virus. Nat Med 2005; 11: 740-747.

36 Meseda CA, Garcia AD, Kumar A, Mayer AE, Manischewitz J, King LR et al. Enhanced immunogenicity and protective effect conferred by vaccination with combinations of modified vaccinia virus Ankara and licensed smallpox vaccine Dryvax in a mouse model. Virology 2005; 339: 164-175.

37 Amanna IJ, Carlson NE, Slifka MK. Duration of humoral immunity to common viral and vaccine antigens. N Engl J Med 2007; 357: 1903-1915.

38 Dorner T, Radbruch A. Antibodies and B cell memory in viral immunity. Immunity 2007; 27: 384-392.

39 Hammarlund E, Lewis MW, Hansen SG, Strelow LI, Nelson JA, Sexton GJ et al. Duration of antiviral immunity after smallpox vaccination. Nat Med 2003; 9: 1131-1137.

40 Jackson RJ, Maguire DJ, Hinds LA, Ramshaw IA. Infertility in mice induced by a recombinant ectromelia virus expressing mouse zona pellucida glycoprotein 3. Bio Reprod 1998; 58: 152-159.

41 Scalzo AA, Farrell HE, Karupiah G. Techniques for studying murine natural killer cells in defense against viral infection. Methods Mol Biol 2000; 121: 163-177.

42 Coulibaly S, Bruhl P, Mayrhofer J, Schmid K, Gerencer M, Falkner FG. The nonreplicating smallpox candidate vaccines defective vaccinia Lister (dVV-L) and modified vaccinia Ankara (MVA) elicit robust long-term protection. Virology 2005; 341: 91-101.

43 Ferrier-Rembert A, Drillien R, Tournier JN, Garin D, Crance JM. Short- and long-term immunogenicity and protection induced by non-replicating smallpox vaccine candidates in mice and comparison with the traditional 1st generation vaccine. Vaccine 2008; 26: 1794-1804. 